\title{
Perceptions and Impact of the 2017 Canadian Guideline for Opioid Therapy and Chronic Noncancer Pain: A Cross-Sectional Study of Canadian Physicians
}

\author{
Jason W. Busse $\mathbb{D}^{1,2,3,4}$ Joyce Douglas, ${ }^{5}$ Tara S. Chauhan, ${ }^{5}$ Bilal Kobeissi, ${ }^{5}$ \\ and Jeff Blackmer \\ ${ }^{1}$ Department of Anesthesia, McMaster University, Hamilton, ON, Canada \\ ${ }^{2}$ Michael G. DeGroote Institute for Pain Research and Care, McMaster University, Hamilton, ON, Canada \\ ${ }^{3}$ Department of Health Research Methods, Evidence and Impact, McMaster University, Hamilton, ON, Canada \\ ${ }^{4}$ Michael G. DeGroote Centre for Medicinal Cannabis Research, McMaster University, Hamilton, ON, Canada \\ ${ }^{5}$ Canadian Medical Association, Ottawa, ON, Canada
}

Correspondence should be addressed to Jason W. Busse; bussejw@mcmaster.ca

Received 30 July 2019; Accepted 25 November 2019; Published 17 February 2020

Academic Editor: Elena K. Enax-Krumova

Copyright ( $\odot 2020$ Jason W. Busse et al. This is an open access article distributed under the Creative Commons Attribution License, which permits unrestricted use, distribution, and reproduction in any medium, provided the original work is properly cited.

Background. Physician adherence to guideline recommendations for the use of opioids to manage chronic pain is often limited. Objective. In February 2018, we administered a 28-item online survey to explore perceptions of the 2017 Canadian guideline for opioid therapy and chronic noncancer pain and if physicians had altered practices in response to recommendations. Results. We invited 34,322 Canadian physicians to complete our survey, and 1,128 responded for a response rate of 3\%; 687 respondents indicated they prescribed opioids for noncancer pain and answered survey questions about the guideline and their practice. Almost all were aware of the guideline, $94 \%$ had read the document, and $89 \%$ endorsed the clarity as good or excellent. The majority ( $86 \%$ ) felt the guideline was feasible to implement, but $66 \%$ highlighted resistance by patients, and $63 \%$ the lack of access to effective nonopioid treatment as barriers. Thirty-six percent of respondents mistakenly believed the guideline recommended mandatory tapering for patients using high-dose opioid therapy ( $\geq 90 \mathrm{mg}$ morphine equivalent per day), and $58 \%$ felt they would benefit from support for opioid tapering. Seventy percent of respondents had changed practices to align with guideline recommendations, with $51 \%$ engaging some high-dose patients in opioid tapering and $43 \%$ reducing the number of new opioid starts. Conclusion. There was high awareness of the 2017 Canadian opioid guideline among respondents, and preliminary evidence that recommendations have changed practice to better align with the evidence. Ongoing education is required to avoid the misunderstanding that opioid tapering is mandatory, and research to identify effective strategies to manage chronic noncancer pain is urgently needed.

\section{Introduction}

Canada is the world's second largest per capita prescriber of opioids [1], and escalating rates of opioid overdoses and deaths [2], in conjunction with increased recognition of the limited effectiveness of opioids for chronic noncancer pain [3], have prompted concerns. In May 2017, the updated Canadian guideline for opioids for chronic noncancer pain was published in the Canadian Medical Association Journal (CMAJ) to promote evidence-based prescribing [4].
However, prior studies have found limited adherence by physicians to guideline recommendations for the use of opioids to manage chronic pain $[5,6]$, explained in part by lack of awareness and familiarity with guidelines [7, 8].

The 2017 guidelines were supported by many Canadian medical organizations, including members of the Pan-Canadian Collaborative for Improved Opioid Prescribing (e.g., the Canadian Medical Association (CMA), the Association of Faculties of Medicine of Canada, the College of Family Physicians of Canada, the Federation of Medical Regulatory 
Authorities of Canada, and the Royal College of Physicians and Surgeons of Canada) [9]. To increase awareness, the Collaborative included a supplement in CMAJ in June 2017 which summarized the guideline [10]. In 2018, the CMA, in partnership with McMaster University, surveyed Canadian physicians to determine their perceptions of the $2017 \mathrm{Ca}$ nadian opioid guideline, and whether they had made changes in their management of chronic noncancer pain patients as a result of the guideline's recommendations.

\section{Materials and Methods}

Through a partnership between McMaster University and the CMA and with the assistance of pain physicians, methodologists, and members of the Pan-Canadian Collaborative for Improved Opioid Prescribing, we developed a 28-item English and French-language questionnaire to examine awareness and perceptions of the 2017 Canadian opioid guideline among Canadian physicians who prescribe opioids for chronic noncancer pain. Respondents were also asked about their preferences for a traditional print format or an online version through MAGICapp (https://app. magicapp.org/app\#/guidelines), an online platform that provides guidelines in an interactive, multilayered format. We further queried if respondents had made any changes to their practices in response to guideline recommendations. We tested the questionnaire with members of the collaborative, and changes were made to include questions regarding supports needed by physicians. We pretested the final questionnaire on a group of 3 physicians who prescribed opioids for chronic noncancer pain, who also commented on its clarity and comprehensiveness and on the time required to complete it (5-8 minutes). No further modifications were suggested by pretest participants (Appendix 1). The Hamilton Integrated Research Ethics Board (HiREB) approved our survey for dissemination without ethics review.

The CMA distributed the questionnaire, on February 1, 2018 , by emailing to a random sample of 34,322 of their members. Physicians were provided with a disclosure letter detailing the intent of the survey and explicit instructions that they could choose not to complete the survey or withdraw at any time. The survey was made available for one month, and three reminder emails were sent at one-week intervals during the study period. Survey respondents were anonymized with the use of a unique identifying number assigned to each participant. We generated frequencies for all collected data and reported categorical data as proportions.

\section{Results}

Of 34,322 physicians approached to complete our survey, 1,128 responded for a response rate of $3 \%$. Six hundred and eighty-seven respondents, who indicated they prescribed opioids for noncancer pain, were eligible to complete specific questions about the guideline and their practice and chose to do so. Approximately half of the eligible respondents were under 55 years of age, 59\% were male (394 of 668), and most were family physicians (68\%; 463 of 678) practicing in
Ontario (41\%; 273 of 667). Forty-two percent (282 of 676) reported that $>10 \%$ of their patients sought care for chronic noncancer pain, and 36\% (214 of 596) prescribed opioids to $>10 \%$ of their patients (Table 1 ).

3.1. Awareness of the 2017 Canadian Opioid Guideline. Almost all respondents were aware of the 2017 Canadian opioid guideline $(92 \% ; 630$ of 686$)$ and had read it thoroughly $(45 \% ; 280$ of 626$)$ or in part $(50 \% ; 310$ of 626$)$. Similar proportions had read the print (39\%; 229 of 588) and online MAGICapp (43\%; 250 of 588) versions; 19\% (109 of 588) had read both. The large majority of respondents endorsed both print and MAGICapp formats as good or excellent (82\% for both); however, twice as many endorsed the online MAGICapp version as excellent (18\% vs. $9 \%)$.

Eighty-nine percent of respondents agreed the clarity of the guideline was excellent $(17 \%$; 93 of 539$)$ or good $(72 \%$; 387 of 539). Some respondents (36\%; 195 of 538) felt the 2017 Canadian opioid guideline had advantages over competing guidelines, whereas $49 \%$ (262 of 538) were unsure and $12 \%$ disagreed (63 of 538). Those who reported advantages most often highlighted its national scope (59\%; 114 of 195) and specific guidance (53\%; 103 of 195). Sixty percent agreed the 2017 Canadian opioid guideline was evidencebased; $32 \%$ were unsure, and $8 \%$ disagreed. (Table 2) When asked if the guideline mandated tapering all patients prescribed high-dose opioids for chronic noncancer pain to $<90 \mathrm{mg}$ morphine equivalent per day (MED), 51\% (269 of 529 ) disagreed, $36 \%$ (193 of 529) agreed, and 13\% (67 of 529) were unsure.

3.2. Barriers and Facilitators of Guideline Implementation. Eight-six percent of respondents felt the guideline was either very feasible (15\%; 85 of 553) or somewhat feasible (70\%; 389 of 553) to implement. Of the $14 \%$ (79 of 553) that felt the guideline was not feasible to implement, the most common concerns were that recommendations were too restrictive regarding the use of opioids $(60 \% ; 47$ of 79$)$ or that recommendations were impractical (58\%; 46 of 79).

Most respondents acknowledged resistance by patients (66\%; 388 of 590), financial barriers to nonpharmacologic treatment $(63 \% ; 374$ of 590$)$, and lack of availability of nonpharmacologic treatment $(63 \% ; 374$ of 590$)$ as implementation challenges. Many respondents noted inadequate time to manage complex cases $(46 \% ; 273$ of 590$)$, lack of access to addiction management services (45\%; 266 of 590), and that it was unrealistic for some of their high-opioid dose legacy patients to engage in tapering (41\%; 243 of 590) (Table 3). When asked if they would benefit from support to help taper high-dose opioid patients, 58\% (264 of 452) agreed; 16\% (70 of 452 ) were uncertain, and 26\% (118 of 452) disagreed. Eightytwo respondents did not reply as they reported no patients in their practice that were prescribed $\geq 90 \mathrm{mg}$ MED.

Most respondents felt that increased coverage for chronic pain treatment options by insurers (60\%; 353 of $590)$, and greater availability of chronic pain treatment services (55\%; 323 of 590) would facilitate guideline implementation. Forty percent of respondents (235 of 590) 
TABLE 1: Demographic characteristics of survey responders.

\begin{tabular}{|c|c|}
\hline \multicolumn{2}{|l|}{ Age $(n=674), n(\%)$} \\
\hline$<35$ & $86(13 \%)$ \\
\hline $35-44$ & $114(17 \%)$ \\
\hline $45-54$ & $158(23 \%)$ \\
\hline $55-64$ & $204(30 \%)$ \\
\hline$\geq 65$ & $112(17 \%)$ \\
\hline \multicolumn{2}{|l|}{ Sex, $(n=668), n(\%)$} \\
\hline Male & $394(59 \%)$ \\
\hline Female & $274(41 \%)$ \\
\hline \multicolumn{2}{|l|}{ Training, $(n=678), n(\%)$} \\
\hline Family physician & $463(68 \%)$ \\
\hline Specialist & $215(32 \%)$ \\
\hline \multicolumn{2}{|c|}{ Province or territory of practice, $(n=667),(\%)$} \\
\hline Ontario & $273(41 \%)$ \\
\hline British Columbia & $128(19 \%)$ \\
\hline Alberta & $121(18 \%)$ \\
\hline Manitoba & $36(5 \%)$ \\
\hline Quebec & $29(4 \%)$ \\
\hline Saskatchewan & $29(4 \%)$ \\
\hline Nova Scotia & $29(4 \%)$ \\
\hline New Brunswick & $10(2 \%)$ \\
\hline Other* & $12(2 \%)$ \\
\hline \multicolumn{2}{|l|}{ Practice setting, $(n=680), n(\%)$} \\
\hline Urban/suburban & $484(71 \%)$ \\
\hline Rural/geographically isolated & $100(15 \%)$ \\
\hline Small town & $96(14 \%)$ \\
\hline
\end{tabular}

Proportion of patients seeking care for chronic

noncancer pain, $(n=676), n(\%)$

$<6 \%$

$218(32 \%)$

$6-10 \%$

$176(26 \%)$

$11-20 \%$

$154(23 \%)$

$21-50 \%$

$75(11 \%)$

$51-75 \%$

$26(4 \%)$

$>75 \%$

$27(4 \%)$

Proportion of chronic noncancer pain patients

prescribed opioids $(n=596), n(\%)$

$<6 \%$

$253(42 \%)$

$6-10 \%$

$129(22 \%)$

$11-20 \%$

$78(13 \%)$

$21-50 \%$

$84(14 \%)$

$51-75 \%$

$27(5 \%)$

$>75 \%$

$25(4 \%)$

*Newfoundland ( $n=5,0.7 \%)$; Yukon Territory $(n=3,0.4 \%)$; Prince Edward Island $(n=2,0.3 \%)$; Northwest Territories $(n=2,0.3 \%)$.

indicated that billing incentives were required to encourage physicians to spend more time with their chronic noncancer pain patients. The most requested topics for continuing medical education (CME) among respondents were for assistance managing demanding, resistant, or nonadherent patients on chronic opioids (58\%; 339 of 590) and instruction on nonopioid options for managing chronic noncancer pain $(44 \% ; 262$ of 590) (Table 3). The most desirable formats for continuing medical education were small groups $(31 \% ; 160$ of 509$)$ and online courses or archived videos $(23 \%$; 117 of 509$)$.

3.3. Changes in Practice due to the Guideline. The majority of respondents $(57 \% ; 318$ of 558$)$ agreed there were areas where
TABLE 2: Respondents impressions of the 2017 Canadian opioid guideline.

\begin{tabular}{lc}
\hline \multicolumn{1}{l}{ Awareness of the guideline $(n=686), n(\%)$} & $630(92 \%)$ \\
Yes & $56(8 \%)$ \\
No & \\
\hline Guideline version read $(n=588), n(\%)$ & $229(39 \%)$ \\
Print version only & $250(43 \%)$ \\
Online MAGICapp version only & $109(18 \%)$ \\
Print and online MAGICapp versions & \\
Neither & $29(9 \%)$ \\
\hline Format of the print version $(n=326), n(\%)$ & $239(73 \%)$ \\
Excellent & $31(10 \%)$ \\
Good & $24(7 \%)$ \\
Unsure/do not recall & $3(1 \%)$ \\
Poor & \\
Very poor & $63(18 \%)$ \\
\hline Format of the online MAGICapp version $(n=345)$, & $219(64 \%)$ \\
$n$ (\%) & $38(11 \%)$ \\
Excellent & $23(7 \%)$ \\
Good & $2(1 \%)$ \\
Unsure/do not recall & \\
Poor &
\end{tabular}

Advantages over competing opioid guidelines

$(n=195), n(\%)^{1}$

National in scope

$114(59 \%)$

More specific guidance

$103(53 \%)$

Broadly endorsed

$94(48 \%)$

Better evidence review process

$83(43 \%)$

The 2017 guideline is evidence-based $(n=505), n(\%)$

Strongly agree

$65(13 \%)$

Agree

$240(48 \%)$

Uncertain

$160(32 \%)$

Disagree

$36(7 \%)$

Strongly disagree

$4(1 \%)$

${ }^{1}$ Percentages add up to $>100 \%$ as respondents could endorse more than one option.

their practice had differed from recommendations of the 2017 Canadian opioid guideline, and 79\% said they had $(70 \%)$ or intended $(9 \%)$ to make changes to their practice because of guideline recommendations. Only 16\% (96 of 590) reported they did not intend to make any changes to their practice. Specifically, in response to the guideline, 51\% (301 of 590) of respondents had engaged some of their legacy patients in opioid tapering, and $43 \%$ had reduced the number of new starts of opioid therapy (254 of 590). Approximately a third (36\%; 215 of 590 ) had reduced the number of opioid tablets they prescribed at one time, and $32 \%$ (191 of 590) had reduced the dose of opioids they prescribed for new starts. A small minority of physicians had either stopped prescribing opioids for chronic noncancer pain $(8 \%$; 46 of 590$)$ or increased their prescribing of opioids for chronic pain (1\%; 5 of 590) (Table 4).

\section{Discussion}

Almost all opioid-prescribing survey respondents were aware of the 2017 Canadian opioid guideline and endorsed its clarity and feasibility of implementation; the majority 
TABLE 3: Barriers and facilitators to implementation of the 2017 Canadian opioid guideline.

\begin{tabular}{|c|c|}
\hline \multicolumn{2}{|l|}{ Implementation challenges $(n=590), n(\%)^{1}$} \\
\hline Reluctance by patients & $388(66 \%)$ \\
\hline Financial barriers to nonpharmacologic treatment alternatives & $374(63 \%)$ \\
\hline Lack of nonpharmacologic treatment alternatives & $371(63 \%)$ \\
\hline Inadequate time to deal with complex cases & $273(46 \%)$ \\
\hline Lack of access to addiction management services & $266(45 \%)$ \\
\hline Unrealistic to taper some high-dose legacy patients to $<90 \mathrm{mg}$ MED & $243(41 \%)$ \\
\hline Lack of access to specialists for support & $218(37 \%)$ \\
\hline Financial barriers to addiction management services & $189(32 \%)$ \\
\hline Need better training in chronic pain management & $127(22 \%)$ \\
\hline Need more training in chronic pain management & $118(20 \%)$ \\
\hline Need better training in addiction management & $114(19 \%)$ \\
\hline \multicolumn{2}{|l|}{ Implementation facilitators $(n=590), n(\%)^{1}$} \\
\hline More chronic pain treatment options covered by insurance & $353(60 \%)$ \\
\hline Greater availability of chronic pain treatment services & $323(55 \%)$ \\
\hline Billing incentives to spend more time with chronic pain patients & $235(40 \%)$ \\
\hline Greater availability of addiction treatment services & $221(38 \%)$ \\
\hline Continuing medical education on opioid deprescribing & $202(34 \%)$ \\
\hline Continuing medical education on nonopioid management of chronic pain & $180(31 \%)$ \\
\hline Real-time access to a prescription monitoring database & $153(26 \%)$ \\
\hline Access to treatment to support behavioral change & $126(21 \%)$ \\
\hline Clinical decision support system integrated into EMRs & $116(20 \%)$ \\
\hline Access to support, such as self-assessments, checklists, and algorithms & $120(20 \%)$ \\
\hline Continuing medical education on opioid prescribing & $109(19 \%)$ \\
\hline Mentorship programs in chronic noncancer pain and addiction & $90(15 \%)$ \\
\hline \multicolumn{2}{|l|}{ Continuing medical education topics of interest $(n=590), n(\%)^{2}$} \\
\hline Managing demanding, resistant, or nonadherent patients on chronic opioids & $339(58 \%)$ \\
\hline Nonopioid options for chronic noncancer pain management & $262(44 \%)$ \\
\hline Strategies to safely taper opioids & $209(35 \%)$ \\
\hline Initiating opioid substitution therapy & $190(32 \%)$ \\
\hline Community programs to reduce opioid addiction and deaths & $117(20 \%)$ \\
\hline Screening, initiating, and monitoring patients on opioid therapy & $106(18 \%)$ \\
\hline Preventing and managing opioid overdoses & $38(6 \%)$ \\
\hline
\end{tabular}

${ }^{1}$ Percentages add up to $>100 \%$ as respondents could endorse more than one option. ${ }^{2}$ Percentages add up to $>100 \%$ as respondents could endorse up to 3 options. EMRs: electronic medical records.

TABle 4: Changes in practice due to the 2017 Canadian opioid guideline.

\footnotetext{
New practice behaviors $(n=590), n(\%)^{1}$

Engaged some legacy patients in opioid tapering Reduced the number of new starts of opioids for chronic noncancer pain

Reduced the number of pills dispensed at one time, for opioid prescriptions

Reduced the dose of opioids for new starts Avoid prescribing opioids for chronic noncancer pain patients with an active substance use disorder Avoid prescribing opioids for chronic noncancer pain patients who also have an active psychiatric disorder, aside from substance use disorder Avoid prescribing opioids for specific patients with chronic noncancer pain based on criteria other than having an active substance use disorder or an active psychiatric disorder

Avoid prescribing opioids for any chronic noncancer pain patients

Prescribe opioids to more patients with chronic noncancer pain

$301(51 \%)$

$254(43 \%)$

$161(27 \%)$

$102(17 \%)$

${ }^{1}$ Percentages add up to $>100 \%$ as respondents could endorse more than one option.
}

agreed the guideline was evidence-based. Resistance from patients and lack of access to effective nonpharmacologic therapy for chronic pain were the main reported barriers to implementation, and most respondents wanted assistance with tapering high-dose opioid patients. Only half of our respondents were confident that the guideline did not mandate tapering for patients prescribed high-dose opioid, and the majority had altered their practices as a result of the guideline, most often involving tapering legacy patients and reducing the number of new starts of opioid therapy for chronic noncancer pain patients.

4.1. Relevant Literature. The 2017 guideline was published with the MAGICapp online platform due to feedback that print versions were cumbersome to use in practice [11], and twice as many respondents rated the MAGICapp version vs. the print version as excellent. The primary reported barrier to guideline implementation was patient reluctance, and the most requested CME topic by respondents was management of difficult patients. Many physicians find patients with chronic noncancer pain challenging [12], and a 2017 qualitative study of family physicians in Ontario, Canada, 
found many did not feel equipped to navigate discussions with chronic pain patients with the result that some avoided prescribing opioids in order to avoid challenging discussions. Some physicians also reported blaming their College or guidelines as a way to escape conflict with patients when pursuing opioid tapering [13].

Physicians are well-trained in prescribing, but not always in deprescribing, and a number of our survey respondents highlighted this challenge; the third most requested CME topic was strategies to safely taper opioids. There is likely a dose-dependent increase in the risk of nonfatal and fatal opioid overdose $[14,15]$. Moreover, low quality evidence suggests that opioid tapering is often successful and may improve patients' pain, function, and quality of life [16, 17]; however, all evidence relates to voluntary tapering. Mandatory opioid tapering is not recommended by the 2017 Canadian opioid guideline [4] and should not be pursued [18], in part because loss of prescription opioids, and resulting withdrawal symptoms may cause some patients to seek out illicit, more dangerous sources [19].

It is noteworthy that approximately a third of respondents to our survey felt the 2017 guideline endorsed mandatory opioid tapering among patients prescribed high-dose opioid therapy, and this may reflect confusion between weak and strong recommendations. Recommendation 9 of the guideline, which suggests tapering opioids to the lowest effective dose for patients prescribed $\geq 90 \mathrm{mg}$ MED, potentially including discontinuation, rather than making no change in opioid therapy, is a weak recommendation. This means that while the majority of informed patients would choose the recommended course of action, an appreciable minority would not. With weak recommendations, clinicians should recognize that different choices will be appropriate for individual patients, and they should help patients arrive at decisions consistent with their values and preferences. The online version of the 2017 Canadian guideline includes patient decision aids for all weak recommendations guideline/8nyb0E).

On October 18, 2019, the College of Physicians and Surgeons of Alberta, HELP Alberta's Pain (a patient advocacy group; https://www.helpalbertaspain.com/), the Alberta Medical Association Section of Chronic Pain, and the Pain Society of Alberta signed an Agreement in Principle to support chronic pain patients prescribed long-term opioid therapy (https://www.painab.ca/uploads/1/0/8/0/ 108066059/aip.pdf). This document, which sites the 2017 Canadian Opioid Guideline for support, advises, in part, that no individual on long-term opioid therapy should be abruptly discontinued. Similar agreements in other provinces may be helpful for re-enforcing this message among prescribers.

Many respondents felt that greater access to effective chronic pain management was required; however, while nonopioid alternatives would be expected to reduce harms associated with long-term opioid use, the incremental benefit for chronic pain sufferers is uncertain. Pharmacologic therapies that have been directly compared with opioids show similar, small effects on pain and function [3].
Moreover, a study of 45,303 US adults with back and neck pain found that although total healthcare expenditures adjusted for inflation increased 65\% from 1997 to 2005, from $\$ 52$ to $\$ 86$ billion/year, age- and sex-adjusted self-reported measures of mental health, physical functioning, work limitations, and social limitations were worse in 2005 than in 1997 [20]. Canada spends approximately \$7.2 billion per year in direct healthcare for chronic noncancer pain [21]; however, from 2003 to 2008, the average funding for pain research in Canada was $\$ 17$ million per year, which represented $<1 \%$ of total funding from the Canadian Institutes of Health Research and $<0.25 \%$ of total health research funding in Canada [22]. It may be that greater investment in research is needed to establish more effective strategies for chronic pain versus simply providing greater access to currently available therapies.

4.2. Strengths and Limitations. Strengths of our study include a comprehensive sampling of Canadian physicians who prescribe opioids for chronic noncancer pain. Moreover, our survey development and administration was consistent with best practices [23]. Our findings are limited by our low response rate $(3 \%)$, which was similar to a prior survey of Canadian physicians' practices regarding opioid management of chronic noncancer pain (710 of 32,000;2\% response rate) [24], and greatly reduces the generalizability of our results.

\section{Conclusions}

There was very high awareness of the 2017 Canadian guideline for opioid therapy and chronic noncancer pain among respondents to our survey, and most physicians reported changing their practices to align with recommendations. Optimizing opioid-prescribing for chronic noncancer pain should reduce opioid-related harms, but confirmation from rigorously conducted observational studies is required. Further education appears needed to ensure appropriate interpretation of weak and strong guideline recommendations, and re-enforcement that forced opioid tapering is inappropriate. The Canadian opioid crisis has highlighted the urgent need for improved management of chronic pain; however, the lack of highly effective therapies suggests that further research is urgently needed versus simply expanding access to existing treatment options.

\section{Data Availability}

The study data used to support the findings of this study are included within the article.

\section{Conflicts of Interest}

The authors declare that they have no conflicts of interest.

\section{Authors' Contributions}

Each author contributed substantially to the conception and design of the article. Jason Busse drafted the initial 
manuscript. Each author revised the manuscript critically for important intellectual content, gave final approval of the version to be published, and agreed to be accountable for all aspects of the work.

\section{Supplementary Materials}

Appendix: Opioid Guideline Survey. (Supplementary Materials)

\section{References}

[1] International Narcotics Control Board (INCB), "Narcotic drugs: estimated world requirements for 2016," INCB, Vienna, Austria, United Nations Publication Sales No. T.16.XI.2, 2016.

[2] Opioid-related harms and deaths in Canada," June 2019, Government of Canada, https://www.canada.ca/en/healthcanada/services/substance-use/problematic-prescription-druguse/opioids/data-surveillance-research/harms-deaths.html.

[3] J. W. Busse, L. Wang, M. Kamaleldin et al., "Opioids for chronic noncancer pain," JAMA, vol. 320 , no. 23, pp. 24482460, 2018.

[4] J. W. Busse, D. N. Juurlink, D. N. Buckley, and G. H. Guyatt, "The authors respond to "inconsistencies in the 2017 Canadian guideline for opioids for chronic noncancer pain"," Canadian Medical Association Journal, vol. 189, no. 39, pp. E1234-E1235, 2017.

[5] L. Khalid, J. M. Liebschutz, Z. Xuan et al., "Adherence to prescription opioid monitoring guidelines among residents and attending physicians in the primary care setting," Pain Medicine, vol. 16, no. 3, pp. 480-487, 2015.

[6] V. Martinez, N. Attal, B. Vanzo et al., "Adherence of French GPs to Chronic Neuropathic Pain Clinical Guidelines: results of a Cross-Sectional, Randomized, "e" Case-Vignette Survey," PLoS One, vol. 9, no. 4, Article ID e93855, 2014.

[7] E. E. Krebs, A. A. Bergman, J. M. Coffing, S. R. Campbell, R. M. Frankel, and M. S. Matthias, "Barriers to guidelineconcordant opioid management in primary care-A qualitative study," The Journal of Pain, vol. 15, no. 11, pp. 1148-1155, 2014.

[8] M. D. Cabana, C. S. Rand, N. R. Powe et al., "Why don't physicians follow clinical practice guidelines?" JAMA, vol. 282, no. 15, pp. 1458-1465, 1999.

[9] College of Family Physicians of Canada, "About the Pan Canadian collaborative," CFPC, Ottawa, Canada, July 2019, https://www.cfpc.ca/chronic-non-cancer-pain-managementopioid-resources-about/.

[10] Canadian Medical Association (CMA), "2017 Canadian opioid prescribing guideline," CMA, Ottawa, Canada, July 2017, https://www.cma.ca/sites/default/files/2018-11/2139_ cma_opioid_poster_en.pdf.

[11] Y. Chang, K. L. Zhu, I. D. Florez et al., "Attitudes toward the Canadian guideline for safe and effective use of opioids for chronic non-cancer pain: a qualitative study," Journal of Opioid Management, vol. 12, pp. 377-387, 2016.

[12] M. Sharpe, R. Mayou, V. Seagroatt et al., "Why do doctors find some patients difficult to help?" QJM: An International Journal of Medicine, vol. 87, no. 3, pp. 187-193, 1994.

[13] L. Desveaux, M. Saragosa, N. Kithulegoda, and N. M. Ivers, "Understanding the behavioural determinants of opioid prescribing among family physicians: a qualitative study," BMC Family Practice, vol. 20, no. 1, p. 59, 2019.
[14] K. M. Dunn, K. W. Saunders, C. M. Rutter et al., "Opioid prescriptions for chronic pain and overdose," Annals of Internal Medicine, vol. 152, no. 2, pp. 85-92, 2010.

[15] E. Kaplovitch, T. Gomes, X. Camacho, I. A. Dhalla, M. M. Mamdani, and D. N. Juurlink, "Sex differences in dose escalation and overdose death during chronic opioid therapy: a population-based cohort study," PLoS One, vol. 10, no. 8, Article ID e0134550, 2015.

[16] J. W. Frank, T. I. Lovejoy, W. C. Becker et al., "Patient outcomes in dose reduction or discontinuation of long-term opioid therapy," Annals of Internal Medicine, vol. 167, no. 3, pp. 181-191, 2017.

[17] B. D. Darnall, M. S. Ziadni, R. L. Stieg, I. G. Mackey, M.-C. Kao, and P. Flood, "Patient-centered prescription opioid tapering in community outpatients with chronic pain," JAMA Internal Medicine, vol. 178, no. 5, pp. 707-708, 2018.

[18] B. D. Darnall, D. Juurlink, R. D. Kerns et al., "International stakeholder community of pain experts and leaders call for an urgent action on forced opioid tapering," Pain Medicine, vol. 20, pp. 429-433, 2019.

[19] J. W. Busse, D. Juurlink, and G. H. Guyatt, "Addressing the limitations of the CDC guideline for prescribing opioids for chronic noncancer pain," Canadian Medical Association Journal, vol. 188, no. 17-18, pp. 1210-1211, 2016.

[20] B. I. Martin, R. A. Deyo, S. K. Mirza et al., "Expenditures and health status among adults with back and neck problems," JAMA, vol. 299, no. 6, pp. 656-664, 2008.

[21] M.-E. Hogan, A. Taddio, J. Katz, V. Shah, and M. Krahn, "Incremental health care costs for chronic pain in Ontario, Canada," Pain, vol. 157, no. 8, pp. 1626-1633, 2016.

[22] M. E. Lynch, D. Schopflocher, P. Taenzer, and C. Sinclair, "Research funding for pain in Canada," Pain Research and Management, vol. 14, no. 2, pp. 113-115, 2009.

[23] K. E. A. Burns, M. Duffett, M. E. Kho et al., "A guide for the design and conduct of self-administered surveys of clinicians," Canadian Medical Association Journal, vol. 179, no. 3, pp. 245-252, 2008.

[24] M. J. Allen, M. M. Asbridge, P. C. Macdougall, A. D. Furlan, and O. Tugalev, "Self-reported practices in opioid management of chronic noncancer pain: a survey of Canadian family physicians," Pain Research and Management, vol. 18, no. 4, pp. 177-184, 2013. 